\title{
Foreword to special issue on wireless ad hoc, sensor and mesh networks
}

\author{
Isaac Woungang
}

Published online: 27 January 2010

(C) Springer Science+Business Media, LLC 2010

The last few years have experienced a steep growth in research in the area of infrastructure-less networks such as wireless ad hoc, sensor and mesh networks. Even though these networks hold great promises for our future, there are several challenges that need to be addressed. This special issue focuses on research relating to different experimental, simulation, algorithmic and theoretical aspects of mobile ad hoc networks, sensor networks, mesh networks, and other related areas.

Limited lifetime is one of the most prevalent concerns in wireless sensor networks (WSNs). In the paper "Modeling the Energy Cost of a Fully Operational Wireless Sensor Network", W. Dargie, C. Xiaojuan, and M. K. Denko analyze the cost associated with self-organization in WSNs, and present a realistic analytic model to determine where the bulk of the energy budget is consumed in WSNs.

Efficient routing and QoS provisioning mechanisms to support the diverse applications are part of the Mobile ad hoc networks (MANETs) requirements. To that effect, N. Zhang and A. Anpalagan in their paper "Sensitivity of SWAN QoS Model in MANETs with Proactive and Reactive Routing: A Simulation Study" confirm that a QoS model referred to as Stateless wireless ad hoc networks (SWAN) model can improve the performance of the MANET in terms of end-to-end delay when there is large traffic in the network.

In Mobile WiMAX networks based on IEEE 802.16e standard, efficient schemes of scheduling and resource allocation that could improve the system performance and ca-

I. Woungang $(\bowtie)$

Department of Computer Science, Ryerson University, Toronto, ON, Canada

e-mail: iwoungan@scs.ryerson.ca pacity are desirable. In the paper "Downlink Resource Allocation Strategies for OFDMA Based Mobile WiMAX", T. Ali-Yahiya, A. L. Beylot, and G. Pujolle, propose two resource allocation strategies, referred to as Adaptive Slot Allocation and Reservation-Based Slot Allocation, for allocating higher layer data to the basic resource allocation units of OFDMA frames. These are shown to achieve good capacity gains for all types of service flows.

Mobile Ad hoc networks are very popular, but more work is required so that they can themselves guarantee network management role and attribute connection parameters provisioning. In the paper "Distributed Address Auto Configuration Protocol for MANET Networks", S. M. Gammar, E. Amine, and F. Kamoun propose a distributed mechanism for auto configuration of MANET, which can be used to handle the network merge and partitioning.

In wireless ad-hoc multi-hop networks, the hop count shift problem in realistic time-varying and lossy radio channels is an open issue. In the paper "The Hop Count Shift Problem and Its Impacts on Protocol Design in Wireless Ad Hoc Networks", B. Wang, H. B. Lim, D. Ma, and C. Fu, propose a strategy to combat the hop count shift problem and conduct simulations to show its effectiveness.

A major challenge WSN deployment is to improve energy-efficiency and lifetime of nodes while providing a reliable communication. M. E. Pellenz, R. D. Souza, and M. S. P. Fonseca in their paper "Error Control Coding in Wireless Sensor Networks: Trade-off between Transmission and Processing Energy Consumption" demonstrate that the use of error correcting convolutional codes in WSNs can substantially increase the node and network lifetime.

In IEEE 802.11 multi-hop ad hoc wireless networks, the current TCP is not able to distinguish corruption losses from packet loss events. To that effect, R. S. Cheng, D. J. Deng, Y. M. Huang, L. Huang, and H. C. Chao, in their paper 
"Cross-Layer TCP with Bitmap Error Recovery Scheme in Wireless Ad Hoc Networks" propose an error recovery mechanism based on coordination of TCP and IEEE 802.11 MAC protocols.

The transmission of multimedia streams over wireless multimedia sensor networks (WMSNs) is a challenging issue due to the limited transmission bandwidth and power resource of sensor nodes. In their paper "TPGF: Geographic Routing in Wireless Multimedia Sensor Networks", L. Shu, Y. Zhang, L. T. Yang, Y. Wang, M. Hauswirth, and N. Xiong, propose a Two-Phase geographic Greedy Forwarding routing algorithm to facilitate the multimedia streaming data transmission in WMSNs.

With the advent of mobile technologies which provide advanced access service to the Internet, security issues in mobile networks are increasingly important. J.H. Park and Q. Jin in their paper "Effective Session Key Distribution for Secure Fast Handover in Mobile Networks" propose a session key distribution mechanism that reflects the properties of mobile networks. In this regards, the stream control transmission protocol is used as a mean to establish the dynamic address configuration for mobile nodes.

In a wireless network, the throughput of a node depends on the interaction between the Physical layer (PHY) and the Medium Access Control sub-layer (MAC). With a contention based MAC, this relationship is not trivial. In their paper "Performance Analysis of Unslotted CSMA/CA in Wireless Networks", B. Lauwens, B. Scheers, and A. V. de Capelle have studied this relation and proposed a novel analytical model for the saturation throughput of $\mathrm{N}$ contending nodes in a wireless network.

Wireless mesh networks (WMNs) are very popular, but more work is required to alleviate network congestion and improve network utilization in multihop WMNs. C. C. Hu, Y. L. Kuo, C. Y. Chiu, and Y. M. Huang in their paper "Maximum Bandwidth Routing and Maximum Flow Routing in Wireless Mesh Networks" propose an efficient loadbalancing routing protocol in a multi-hop WMN. Further to this contribution, they develop heuristic algorithms to address the problem of determining a maximum bandwidth path and that of determining a maximum flow, in a multihop WMN.

Data gathering is an important challenge for sensor network applications. S. Kwon, J. Shin, J. Ko, and C. Kim in their paper "Distributed and Localized Construction of Routing Structure for Sensor Data Gathering" address this problem. They propose a framework for tree construction, in which the node status information is exchanged locally and a decision based on the local information leads to the construction of a routing tree for data gathering.

With the advancement of wireless sensor network (WSN) applications, localization of nodes in WSNs is a new emerging research challenge. To that effect, N. Irfan, M. Bolic, M. C. E. Yagoub, and V. Narasimhan in their paper "NeuralBased Approach for Localization of Sensors in Indoor Environment" propose a new MLP neural networks-based model to accurately determine the location of the mobile sensor node.

Designing routing schemes that would successfully operate in the presence of adversarial environments in Mobile Ad Hoc Networks (MANETs) is a challenging issue. In their paper "Fault-Tolerant Routing In Adversarial Mobile Ad Hoc Networks: An Efficient Route Estimation Scheme For Non-Stationary Environments", B. J. Oommen and S. Misra propose a new fault-tolerant routing scheme that invokes a stochastic learning-based weak estimation procedure to enhance a route estimation phase, which, in turn, is incorporated in a route selection phase.

Channel assignment problems in multi-radio wireless mesh networks (WMNs) have been shown to be NP hard in various scenarios in the literature. In their paper "Feasibility of optimally assigning channels by exhaustive search in commercial multi-radio wireless mesh networks", W. Xie, Y. J. Zhang, M. L. Sichitiu, L. Fu, and Y. Yao have estimated the theoretical runtime of determining the optimal channel assignment by exhaustive search and validate it through experiments. This work further provides some guidelines for algorithm design and network planning in commercial wireless mesh networks.

I hope that readers will enjoy reading these papers and find them valuable. The readers are encouraged to contact the authors, if they need any further clarification regarding their works presented. I take this opportunity to express a few words of my thankfulness. I would like to thank all authors who submitted their papers to this special issue, as well as all reviewers for their dedicated work. I also would like to thank the Editor-in-Chief, Professor Bezalel Gavish, for his support and guidance to my editorial work, Ms. Jackie James, Administrative Assistant to Professor Gavish, for her valuable work in coordinating the prepublication process. Finally, I thank the staff at Springer for their kind help. 